\title{
Polyelectrolytes as New Matrices for Secondary Ion Mass Spectrometry
}

\author{
Yit-Yian Lua, Li Yang, Craig A. Pew, Feng Zhang, \\ W. Jonathan J. Fillmore, R. Todd Bronson, Amarchand Sathyapalan, \\ and Paul B. Savage \\ Department of Chemistry and Biochemistry, Brigham Young University, Provo, Utah, USA
}

Jed D. Whittaker and Robert C. Davis

Department of Physics, Brigham Young University, Provo, Utah, USA

Matthew R. Linford

Department of Chemistry and Biochemistry, Brigham Young University, Provo, Utah, USA

Significant enhancements in ion yields in time-of-flight secondary ion mass spectrometry (TOF-SIMS) are observed when water-soluble analytes are mixed with a polyelectrolyte, e.g., poly(diallyldimethylammonium chloride) or poly(sodium 4-styrenesulfonate), and then deposited in the layer-by-layer method on a surface. This previously unobserved effect is demonstrated for 5-chloro-8-methoxyquinoline appended diaza-18-crown-6, 5-(2-aminoethoxy)methyl-5-chloro-8-methoxyquinoline appended diaza-18-crown-6, acridine, 9-anthracenecarboxylic acid, and ferrocenecarboxylic acid. By optical ellipsometry film thicknesses range from ca. 5-20 ̊. X-ray photoelectron spectroscopy shows significantly less analyte in the polyelectrolyte-analyte films than in the neat analytes. However, TOF-SIMS generally shows significant enhancements in ion yields from the polyelectrolyte films compared with either the neat compounds or the compounds solubilized with acid or base and then dried on a surface. These significant enhancements in ion yields also appear to extend to analyte fragments and cationized molecular species. Some enhancement is also observed for dried droplets of analytes mixed with a polyelectrolyte on surfaces. (J Am Soc Mass Spectrom 2005, 16, 1575-1582) ( 2005 American Society for Mass Spectrometry

$\mathrm{M}$ ass spectrometry, including time-of-flight secondary ion mass spectrometry [1] (TOFSIMS), which is arguably the most important analytical technique for chemical analysis of surfaces, is wholly reliant on some type of ionization method to produce ions for detection and mass analysis. This requirement is particularly important for TOF-SIMS because most of the atomic and molecular fragments that are ejected from a surface during ion bombardment are neutral. Hence, to increase spectral quality, improve detection limits, and facilitate data interpretation, methods to enhance ion yields, including the complexation of analytes with other species to produce cations, i.e., cationization, have been actively studied and employed since the inception of SIMS more than thirty years ago [2].

Most ionization/cationization methods that are encountered in TOF-SIMS involve the formation of quasi-

Published online August 8, 2005

Address reprint requests to Dr. M. R. Linford, Department of Chemistry and Biochemistry, Brigham Young University, Provo, UT 84602, USA. E-mail: mrlinford@chem.byu.edu molecular ions from which analyte masses can be easily derived. The most frequently encountered are [2]:

1. Protonation and deprotonation to form $[\mathrm{M}+\mathrm{H}]^{+}$ and $[\mathrm{M}-\mathrm{H}]^{-}$[3]. Proton gain and loss occur most frequently with basic and acidic groups, respectively.

2. Loss of small functional groups, e.g., formation of $\left[\mathrm{M}-\mathrm{CH}_{3}\right]^{ \pm}$or $[\mathrm{M}-\mathrm{OH}]^{+}$.

3. Addition of alkali metal ions, e.g., $\mathrm{Li}^{+}, \mathrm{Na}^{+}, \mathrm{K}^{+}$, etc., to form $[\mathrm{M}+\text { alkali }]^{+}[4]$.

4. Addition of substrate metal ions, such as silver or gold, to form $\left[\mathrm{M}+\right.$ metall $^{+}[5-9]$.

Polyelectrolytes (PEs) are macromolecules that contain charged or ionizable groups. A positively charged $\mathrm{PE}$ is a polycation and a negatively charged PE is a polyanion. Examples of the former and latter are poly(diallyldimethylammonium chloride), or PDADMAC, and poly(sodium 4-styrenesulfonate), or PSS, respectively, (see Figure 1). Around neutral $\mathrm{pH}$ silanol groups $\left(\mathrm{pK}_{\mathrm{a}} \sim 4\right)$ on glass and quartz are deprotonated to give 


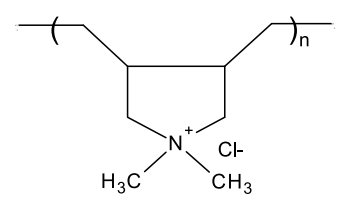

PDADMAC
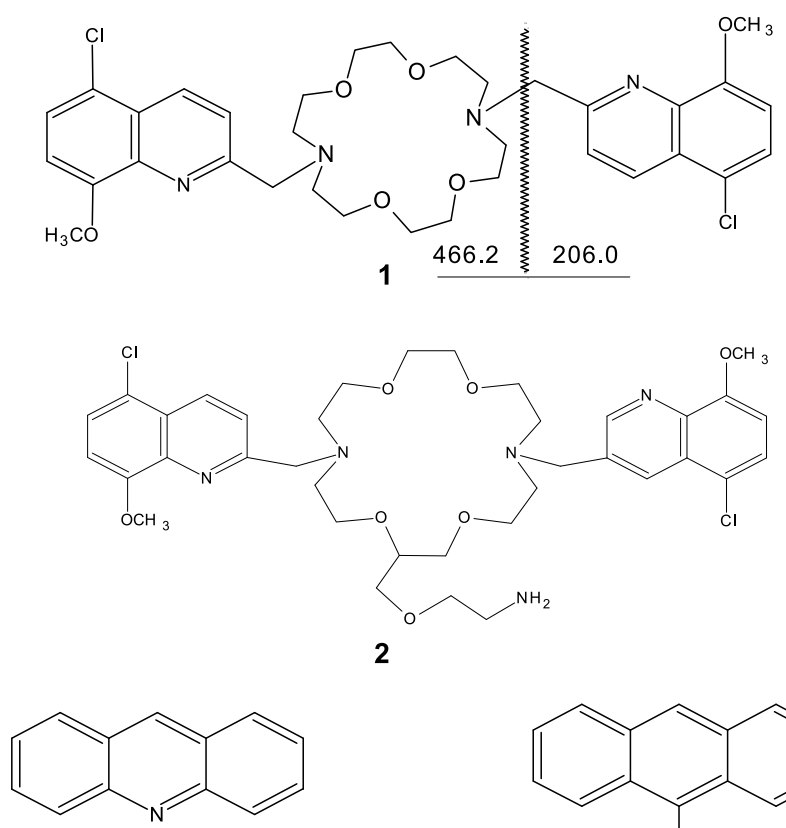

3

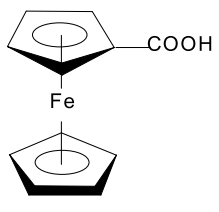

5

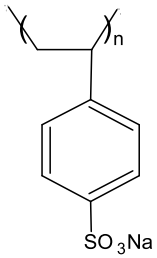

PSS
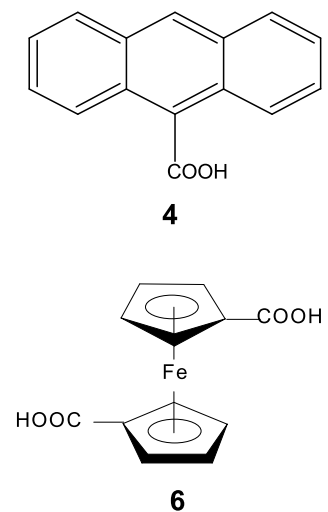

Figure 1. Structures of poly(diallyldimethylammonium chloride) (PDADMAC), poly(sodium 4-styrenesulfonate) (PSS), 5-chloro-8-methoxyquinoline appended diaza-18-crown-6, 1; 5-(2-aminoethoxy) methyl-5-chloro-8-methoxyquinoline appended diaza-18-crown-6, 2; acridine, 3; 9-anthracenecarboxylic acid, 4; ferrocenecarboxylic acid, 5; 1,1'-ferrocenedicarboxylic acid, 6.

their surfaces a negative charge. Various polycations, including PDADMAC, adsorb as thin films onto these negatively charged surfaces, reversing the surface charge to create positively charged layers. Polyanions then spontaneously adsorb onto these positively charged surfaces, again reversing the surface charge. Typical thicknesses for these PE layers are 3 to $25 \AA$. This process may be repeated to build up complex PE multilayer structures [10], which have been characterized by a variety of techniques including TOF-SIMS [11-13]. It has also been shown that a small organic molecule with charged groups can take the place of a PE in a layer-by-layer assembly [14-16]. Premixing of the organic molecule with a PE in solution facilitates ad- sorption of the small molecule [17]. We are not aware of any SIMS work on thin films of PEs that contain small molecules.

Here we demonstrate for the first time that a PE multilayer can significantly enhance the ion yields of complex organic molecules, including 5-chloro-8-methoxyquinoline appended diaza-18-crown-6, 1, 5-(2-aminoethoxy)methyl-5-chloro-8-methoxyquinoline appended diaza-18-crown-6, 2, acridine, 3, 9-anthracenecarboxylic acid, 4, and ferrocenecarboxylic acid, 5 (see Figure 1). Sample preparation is straightforward from aqueous solutions and only employs basic laboratory equipment. For 1-3, sample preparation consists of (1), deposition of a ca. $4 \AA$ PDADMAC layer on the thin native oxide of silicon (16-18 $\AA$ thick), followed by (2), deposition of a mixture of PSS and 1, 2, or 3 in dilute $\mathrm{HCl}$. A lower $\mathrm{pH}$ is necessary to increase the solubility of these molecules in water. For 4 and 5, sample preparation simply consists of deposition of a mixture of PDADMAC and 4 or 5 in dilute $\mathrm{NH}_{4} \mathrm{OH}$ on the thin native oxide of silicon. In this case a higher $\mathrm{pH}$ was advantageous to solubilize the analyte. Before mixing, the PE and analyte stock solutions were clear. After mixing, their mixtures were cloudy, suggesting complexation between the analyte and the PE. Complexation has previously been observed in solution between PEs and water-soluble, charged organic dyes [18, 19]. Some enhancement in ion yield was also observed from a dried droplet of a simple binary solution of an analyte and a polyelectrolyte.

\section{Experimental}

For all preparations, including those made with a single droplet, analyte concentrations were $5 \mathrm{mM}$ and polyelectrolyte concentrations were $10 \mathrm{mM}$ in monomer (these latter concentrations were calculated by dividing the weight of the polymer by the molecular weight of its monomer by the volume of the solution). Solutions were stable without precipitation during the course of the experiments. Immersion times of silicon shards in solutions were $30 \mathrm{~min}$. After immersions, samples were rinsed by dipping three times in vials of Millipore water (Billerica, MA) for $1 \mathrm{~min}$. All solutions containing acidic or basic analytes with low solubility were prepared by mixing the analyte in water, and then adding drops of concentrated $\mathrm{NH}_{4} \mathrm{OH}$ or $\mathrm{HCl}$, respectively, until the analyte dissolved. A small amount of water was finally added to reach the desired volume. TOF-SIMS (performed with an ION-TOF TOF-SIMS IV with monoisotopic $25 \mathrm{keV}^{69} \mathrm{Ga}^{+}$primary ions) was performed as described previously [20]. "Corrected intensities" as provided by the instrument software are reported in this work. $\mathbf{1}$ and $\mathbf{2}$ were synthesized by Savage and coworkers [21]. All other compounds were obtained from Aldrich (Milwaukee, WI) and used as received. Spectroscopic ellipsometry was performed with an M-2000 instrument from J. A. Woollam Co. (Lincoln, NE) AFM was performed with a Dimension 3100 AFM, 
Table 1. Film thicknesses

\begin{tabular}{lcc}
\hline Analyte & $\begin{array}{c}\text { Thickness of } \\
\text { PDADMAC } \\
(\AA)\end{array}$ & $\begin{array}{c}\text { Thickness of } \\
\text { PSS + Analyte (A) } \\
\text { on PDADMAC }\end{array}$ \\
\hline \hline 1 & $3.7 \pm 0.48$ & $15.2 \pm 0.63$ \\
2 & $3.9 \pm 0.37$ & $15.8 \pm 0.77$ \\
3 & $4.5 \pm 0.15$ & $14.5 \pm 0.93$ \\
4 & $5.8 \pm 0.30^{*}$ & - \\
5 & $5.5 \pm 0.23^{*}$ & - \\
\hline
\end{tabular}

*Analytes were mixed with PDADMAC prior to deposition.

equipped with a motorized optical microscope, and a motorized stage. XPS was performed with an SSX-100 $X$-ray photoelectron spectrometer with a monochromatic $\mathrm{Al} \mathrm{K} \alpha$ source and a hemispherical analyzer. Because of the thinness of the polyelectrolyte films (see Table 1), it was not necessary to correct for photoelectron attenuation in drawing the conclusions reached in this work. That is, a typical attenuation length of a photoelectron in an organic thin film is $30 \AA$ [22] and the information depth of XPS is about three times that, or roughly $100 \AA$ for organic materials.

\section{Results}

Spectroscopic ellipsometry over a relatively large area of a surface $(0.5-1.0 \mathrm{~cm})$ provides a good average thickness of an organic thin film. For deposition of 1, 2, and 3, Table 1 shows that the PDADMAC films were consistently 3.5 to $4.5 \AA$ thick, and that the PSS-analyte films were 14.5 to $16 \AA$ thick. As might be expected, the PDADMAC-4 and -5 films were a little thicker than the pure PDADMAC films.

While ellipsometry gives precise, average film thicknesses, atomic force microscopy (AFM) provides a better microscopic picture of thin films. Figure 2 shows AFM images of (Figure 2a) the bare silicon $/ \mathrm{SiO}_{2}$ substrate $(0.15 \mathrm{~nm}$ rms roughness), and (Figures $2 \mathrm{~b}$ and $\mathrm{c}$ ) regions of a silicon surface coated with PDADMAC/ PSS-1. After deposition of the PE bilayer AFM reveals an increase in surface roughness $(0.39 \mathrm{~nm} \mathrm{rms})$ and the presence of a few particles, with some regions showing higher surface roughness $(0.72 \mathrm{~nm} \mathrm{rms})$ and a larger number of particles. The majority of these particles originates during film deposition and are most likely attributable to deposition of PSS and $\mathbf{1}$ that had complexed in solution.

X-ray photoelectron spectroscopy (XPS) provides surface elemental compositions of materials. XPS of neat 1 shows the expected elements (see Figure 3a), including substantial chlorine signals (see inset to Figure 3a). In contrast, survey and narrow XPS scans of the PDADMAC/PSS-1 bilayer show the elements that are expected from the polymers and substrate (carbon, oxygen, nitrogen, sulfur, and silicon), but no chlorine can be detected (inset to Figure $3 b$ ). Thus, XPS shows that the number of $\mathbf{1}$ molecules in the PDADMAC/ PSS-1 bilayer is much smaller than in neat $\mathbf{1}$.

TOF-SIMS of the PDADMAC/PSS-1 bilayer shows peaks attributable to the substrate and the PE. The eight largest peaks in decreasing order of area in the positive ion spectrum are attributed to $\mathrm{Si}^{+}, \mathrm{C}_{3} \mathrm{H}_{8} \mathrm{~N}^{+}, \mathrm{C}_{2} \mathrm{H}_{4} \mathrm{~N}^{+}$, $\mathrm{C}_{2} \mathrm{H}_{3}{ }^{+}, \mathrm{SiH}^{+}, \mathrm{CH}_{4} \mathrm{~N}^{+}, \mathrm{CH}_{3}{ }^{+}$, and $\mathrm{SiOH}^{+}$. In addition, TOF-SIMS yields an unusually strong quasi-molecular ion, $(\mathrm{M}+1)^{+}$for 1 , compared with the $(\mathrm{M}+1)^{+}$signal from either a droplet of $\mathbf{1}$ (dissolved in dilute $\mathrm{HCl}$ and dried on a silicon surface), or neat $\mathbf{1}$ (see complete spectrum in Figure 4). The calculated isotope pattern for the $(\mathrm{M}+1)^{+}$ion of $\mathbf{1}$ (see the inset to Figure 5a) is essentially an exact match to the pattern found in Figure 5a. Enhancements similar to that observed for $(\mathrm{M}+1)^{+}$ of $\mathbf{1}$ in the PDADMAC/PSS-1 bilayer are also found for two fragments of 1, which occur at $\mathrm{m} / \mathrm{z} 468$ and 207 (see Figure $5 \mathrm{~b}$ and c). The structure of $\mathbf{1}$ suggests that the $\mathrm{m} / \mathrm{z}$ 468 ion is the $m / z 466$ fragment with two additional hydrogen atoms and that the $m / z 207$ ion is the $m / z 206$
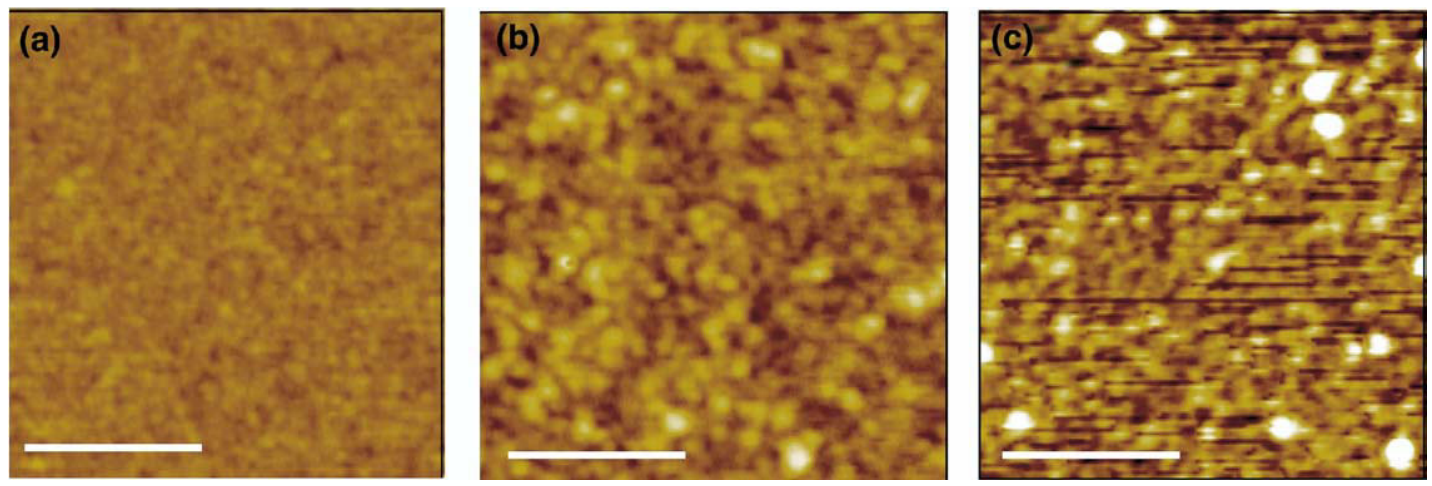

Figure 2. Tapping mode AFM images of bare silicon oxide (the native oxide on a silicon wafer) and PDADMAC/PSS-1 surfaces. Scale bars are $200 \mathrm{~nm}$. Image a was taken on a blank $\mathrm{SiO}_{2}$ surface before deposition and yielded a roughness of $0.15 \mathrm{~nm}$ rms. Image $\mathbf{b}$ was taken after PDADMAC/PSS-1 deposition and yielded a roughness of $0.39 \mathrm{~nm} \mathrm{rms}$. Some regions had a significant density of particles resulting in an increased rms roughness. Image $\mathbf{c}$ was taken in a region with several particulates, the surface roughness was $0.72 \mathrm{~nm}$ but as can be seen by comparison with $\mathbf{B}$, the areas between the large particulates are similar in roughness to Image $\mathbf{B}$. 


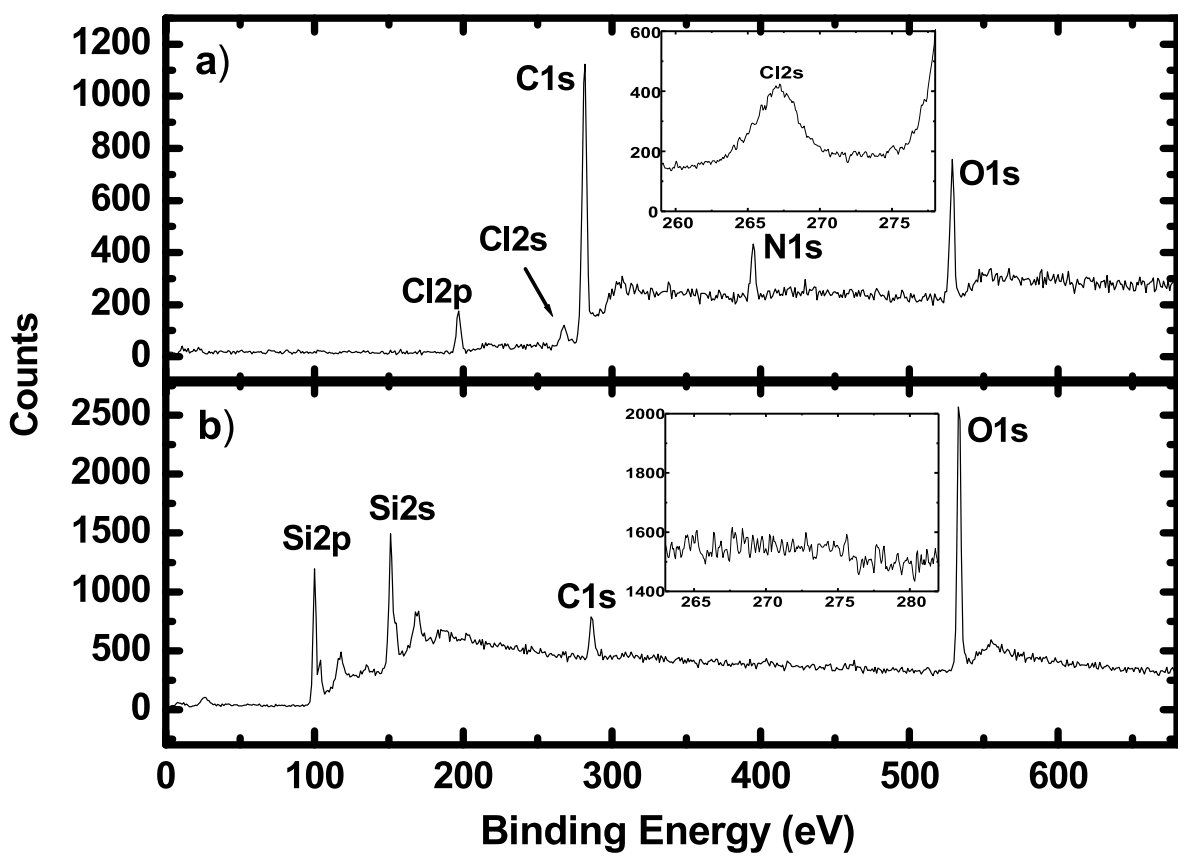

Figure 3. XPS of (a) neat $\mathbf{1}$ and (b) a bilayer of PDADMAC/PSS-1. The insets show the $\mathrm{Cl} 2 \mathrm{~s}$ signal.

fragment with one additional hydrogen atom. The two most basic sites on $\mathbf{1}$ are the $\mathrm{N}$ atoms in the crown ring. Thus, if these two sites were protonated, the $\mathrm{m} / \mathrm{z} 468$ fragment could be created by heterolysis of the $\mathrm{N}-\mathrm{C}$ bond shown in Figure 1. On the other hand, if the $\mathrm{N}$ atom in the aromatic ring were protonated and the

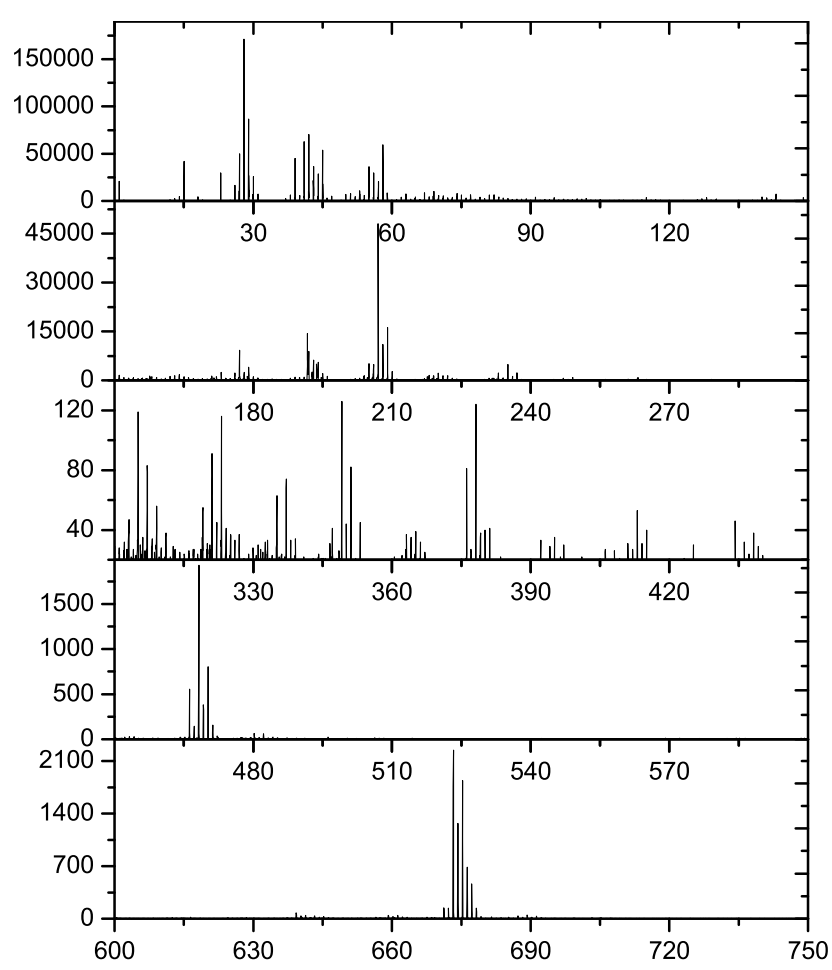

Figure 4. TOF-SIMS spectrum of $\mathbf{1}$ in a polyelectrolyte bilayer from $m / z=0-750$.

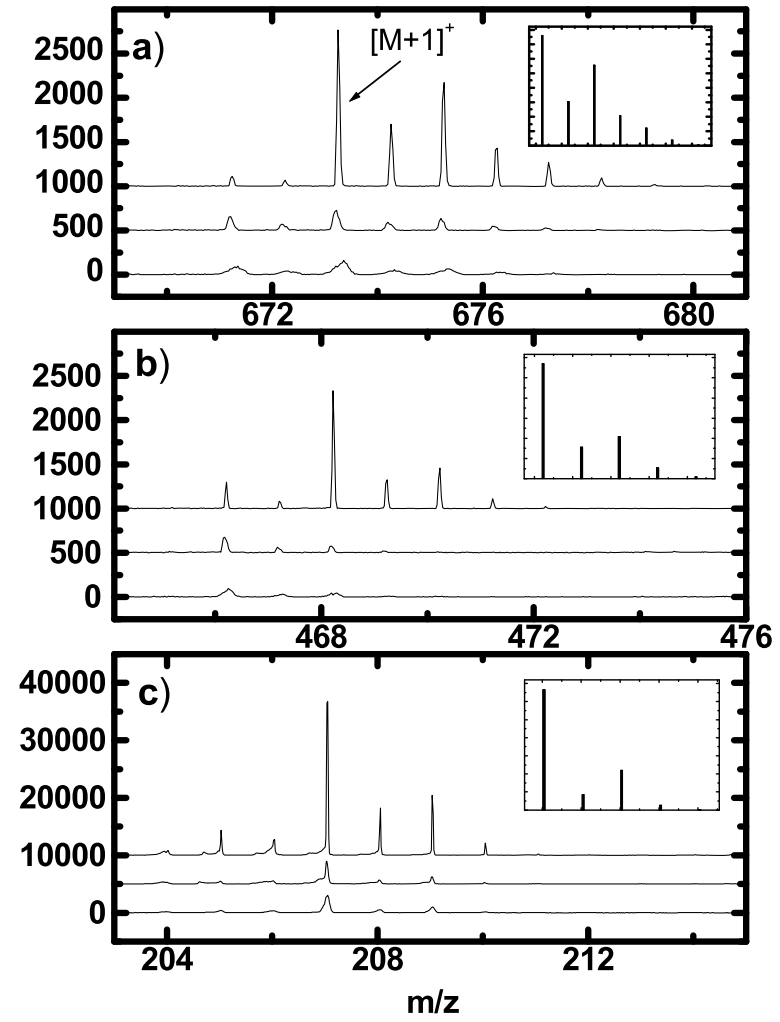

Figure 5. TOF-SIMS spectra (as taken, not normalized or scaled) of the PDADMAC/PSS-1 bilayer (top spectrum in each panel), 1 dissolved in $\mathrm{HCl}$ and then dried on a silicon surface (middle spectrum in each panel), and neat $\mathbf{1}$ (bottom spectrum in each panel). Theoretical intensities of the peaks expected from the different isotopes at $\mathrm{m} / \mathrm{z}$ 673, 468, and 207 are shown as insets. 


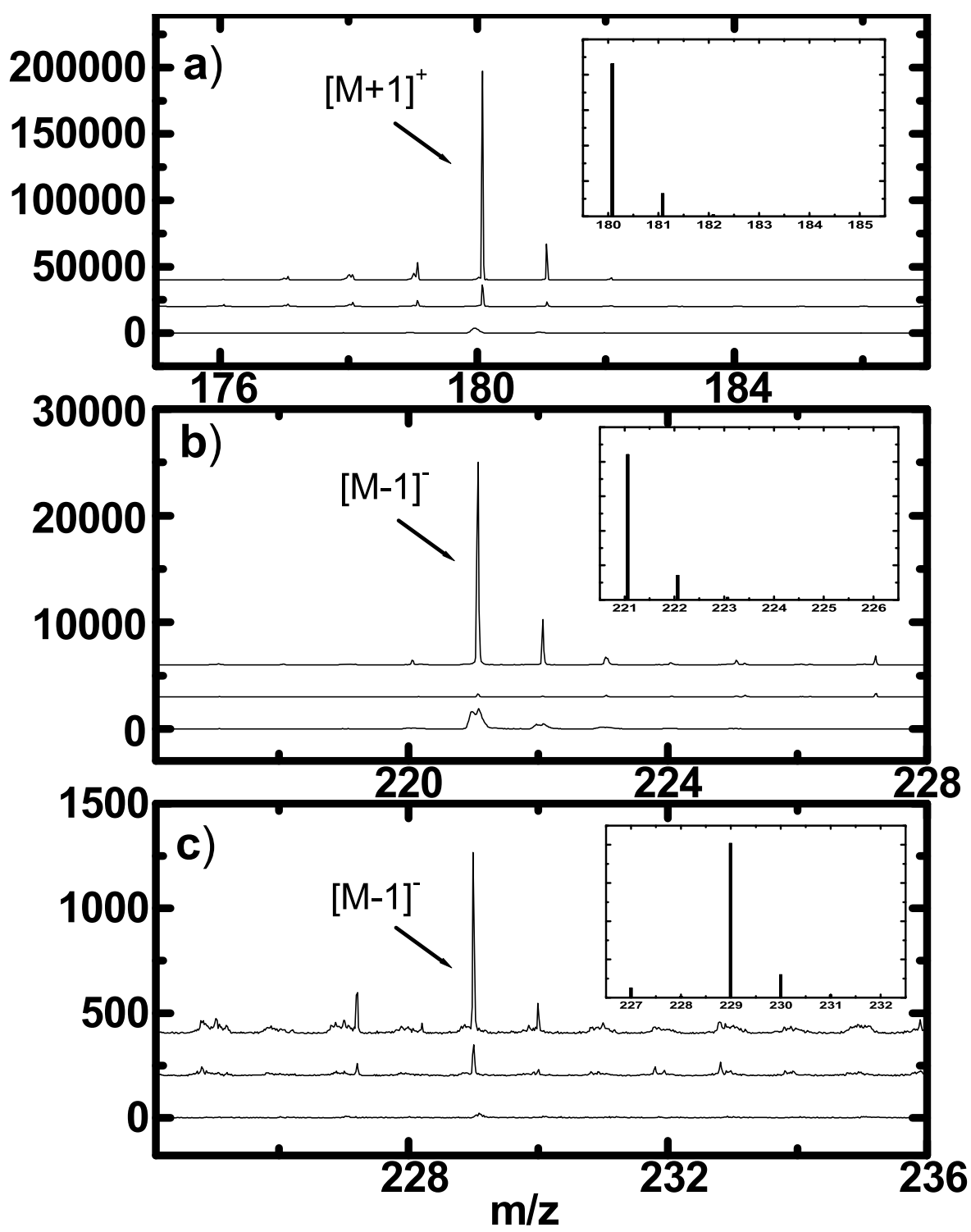

Figure 6. TOF-SIMS spectra (as taken, not normalized or scaled) of quasi-molecular ions of (a) 3, (b) 4, and (c) 5. The PDADMAC/PSS-3 bilayer and the PDADMAC-4 and PDADMAC-5 monolayers are the top spectra in their respective panels. 3 dissolved in $\mathrm{HCl}$ and $\mathbf{4}$ and $\mathbf{5}$ dissolved in $\mathrm{NH}_{4} \mathrm{OH}$, and then allowed to dry on a silicon substrate, are the middle spectra in their respective panels. Neat 3, 4, and 5 are the bottom spectra in their respective panels.

$\mathrm{N}-\mathrm{C}$ bond in $\mathbf{1}$ breaks homolytically as shown in Figure 1, then a $m / z 207$ fragment would be created. The theoretical isotope pattern for the $\mathrm{m} / \mathrm{z} 468$ fragment is essentially an exact match to the experiment. That the agreement is less perfect for the $\mathrm{m} / \mathrm{z} 207$ peak is attributed to the presence of additional components that appears to be in the $m / z 204$ to 210 region.

To test the generality of PEs for enhancing ion yields and cationization of analytes, thin PE films containing 3, 4 , and 5 were studied. The ellipsometric thicknesses of these layers were in good agreement with the results obtained from bilayers containing $\mathbf{1}$ and $\mathbf{2}$ (see Table 1). Figure 6a shows the positive ion TOF-SIMS spectrum of PDADMAC/PSS-3, which shows a significant enhancement in the quasi-molecular ion $(\mathrm{M}+1)^{+}$over controls, as was observed for the PDADMAC/PSS-1 bilayer. A signal corresponding to a quasi-molecular ion of 4 could not be found in the positive ion spectra of PDADMAC-4 or corresponding controls. However, a strong enhancement in ion yield is obtained in the negative ion spectrum for $(\mathrm{M}-1)^{-}$, compared with 4 dissolved in ammonium hydroxide and dried on the surface, or neat 4 (see Figure 6b). Similarly, a strong enhancement in the $(\mathrm{M}-1)^{-}$ion yield was also obtained for PDADMAC-5. Figure 7 shows XPS of neat 5 and the PDADMAC-5 monolayer. Note that, analogous to what was observed in Figure 3, a strong iron signal is obtained from neat 5 , but only a very weak iron signal is obtained from PDADMAC-5. This iron signal would probably not be observable were it not that XPS photoemission in- 


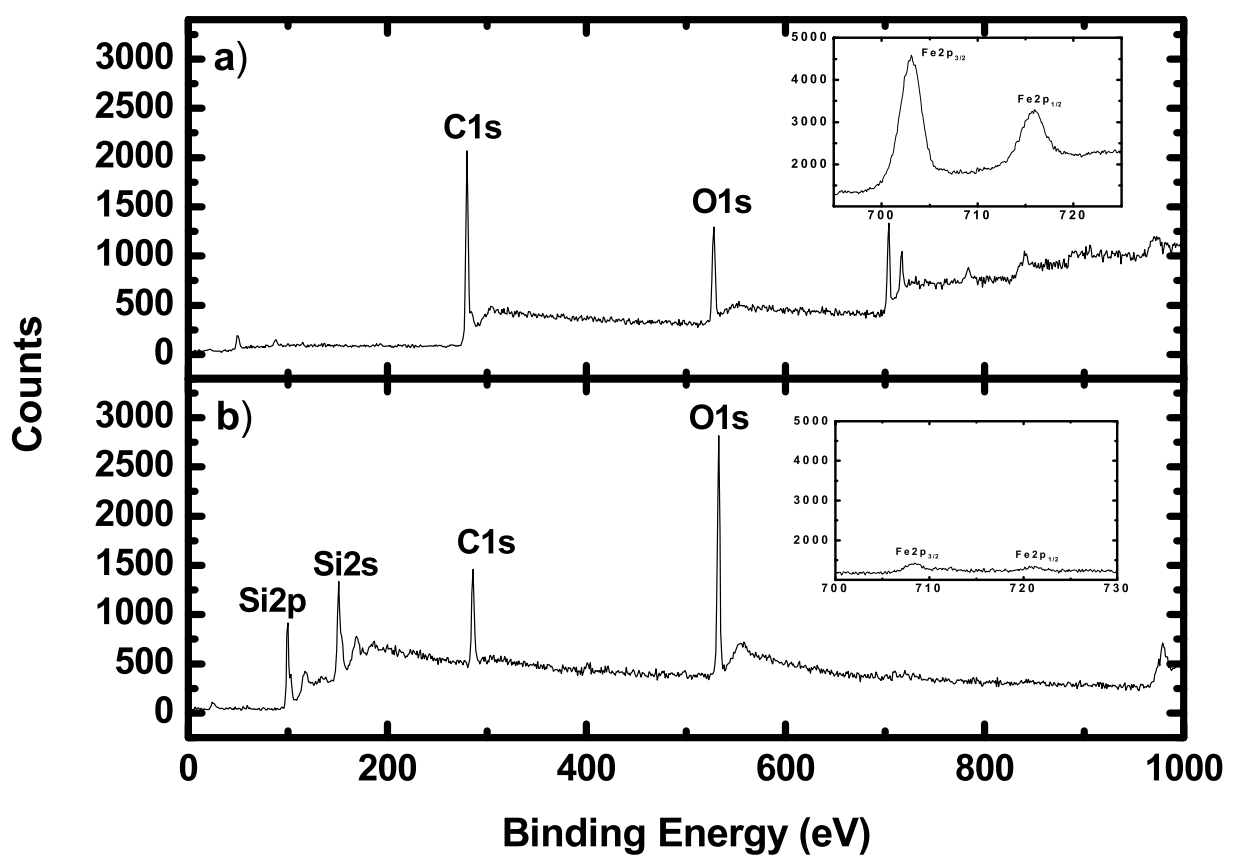

Figure 7. XPS of (a) neat 5 and (b) a monolayer of PDADMAC-5. Insets show the Fe $2 p_{3 / 2}$ and $2 p_{1 / 2}$ peaks.

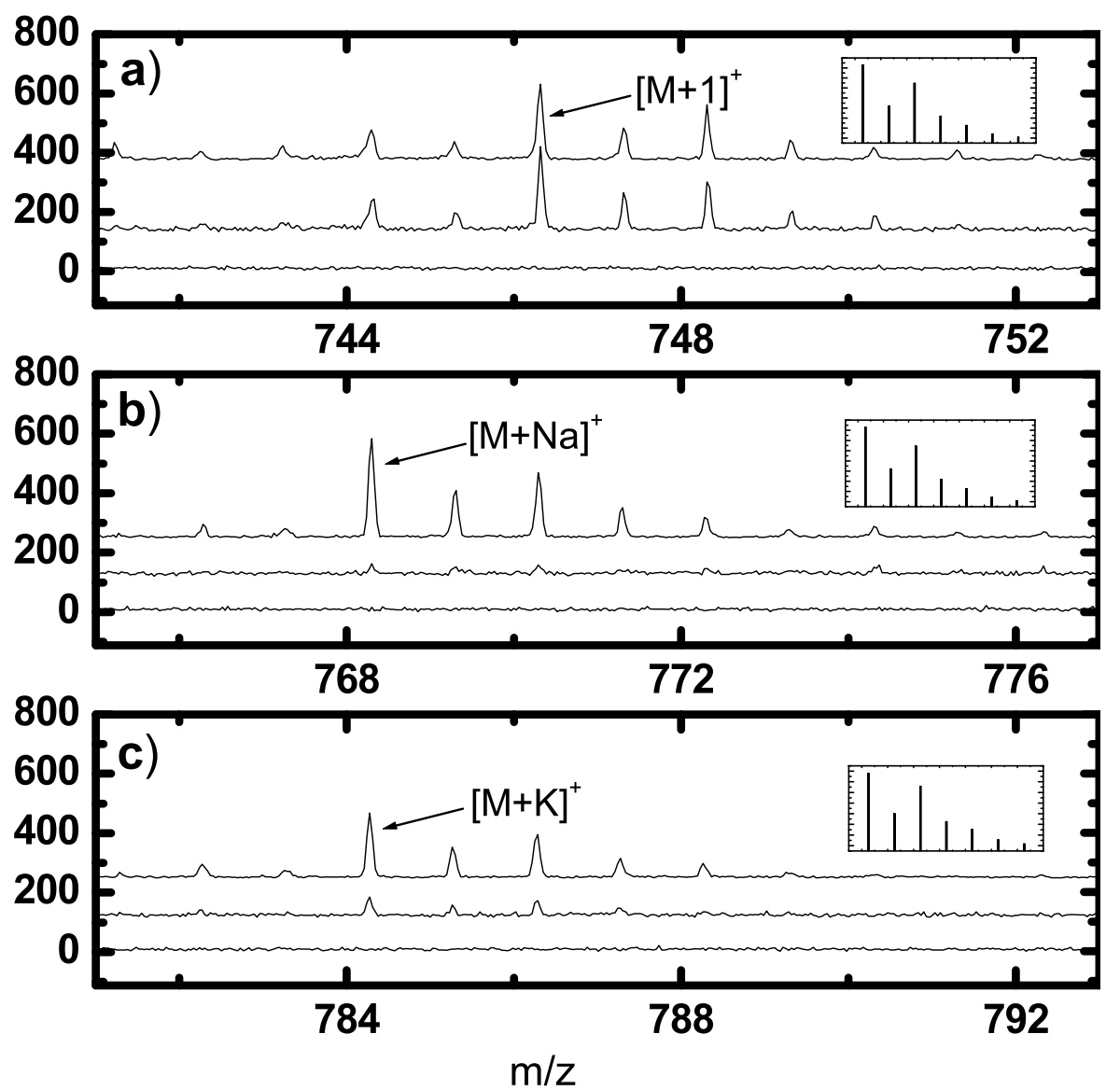

Figure 8. TOF-SIMS spectra (as taken, not normalized or scaled) of the PDADMAC/PSS-2 bilayer (top spectrum in each panel), 2 dissolved in $\mathrm{HCl}$ and then dried on a silicon surface (middle spectrum in each panel), and neat 2 (bottom spectrum in each panel). Theoretical intensities of the peaks expected from the different isotopes at $\mathrm{m} / \mathrm{z} 746,768$, and 784 are shown as insets. 
Table 2. Intensities of quasi-molecular ions from 9-anthracenecarboxylic acid $(\mathrm{M}-1)$, and acridine $(\mathrm{M}+1)$ from TOF-SIMS spectra. Results from two samples of each material are given

\begin{tabular}{|c|c|c|c|c|c|c|c|c|}
\hline \multicolumn{9}{|c|}{ 9-Anthracenecarboxylic Acid, 4 ( $\mathrm{m} / \mathrm{z}:$ 221) } \\
\hline \multirow{3}{*}{$\begin{array}{l}\text { Experiment } \\
\text { Intensity } \\
\text { Resolution }\end{array}$} & \multicolumn{2}{|c|}{$\begin{array}{l}\text { PDADMAC-4 } \\
\text { monolayer }\end{array}$} & \multicolumn{2}{|c|}{$\begin{array}{c}\text { Solution of } 4 \text { in dil. } \\
\mathrm{NH}_{4} \mathrm{OH}\end{array}$} & \multicolumn{2}{|c|}{ Powder 4} & \multicolumn{2}{|c|}{$\begin{array}{l}\text { Solution of } 4 \text { and } \\
\text { PDADMAC }\end{array}$} \\
\hline & 43522 & 42516 & 731 & 998 & 50725 & 53602 & 5991 & 5224 \\
\hline & 9443 & 9787 & 10310 & 10348 & 1202 & 1238 & 10845 & 8916 \\
\hline \multicolumn{9}{|c|}{ Acridine, $3(\mathrm{~m} / \mathrm{z}: 180)$} \\
\hline Experiment & \multicolumn{2}{|c|}{$\begin{array}{l}\text { PDADMAC/PSS-3 } \\
\text { bilayer }\end{array}$} & \multicolumn{2}{|c|}{$\begin{array}{l}\text { Solution of } 3 \text { in dil. } \\
\mathrm{HCl}\end{array}$} & \multicolumn{2}{|c|}{ Powder 3} & \multicolumn{2}{|c|}{$\begin{array}{c}\text { Solution of } 3 \text { and } \\
\text { PSS }\end{array}$} \\
\hline Intensity & 56870 & 49937 & 12173 & 8783 & 10371 & 10820 & 25892 & 23412 \\
\hline Resolution & 10327 & 10349 & 11234 & 12262 & 1465 & 1757 & 10896 & 10794 \\
\hline
\end{tabular}

creases with increasing atomic number: it is more than six times greater for $\mathrm{Fe} 2 \mathrm{p}$ photoelectrons than for $\mathrm{Cl} 2 \mathrm{~s}$ photoelectrons [23]. Thus, we again see that much less analyte in a PE film yields a much stronger TOF-SIMS signal than either the neat analyte or the protonated or deprotonated analyte.

To test the effect of a small change in structure on the ionization/cationization of the PDADMAC/PSS-1 bilayer, a bilayer containing an amine-appended macrocycle, 2, was made. Based on the structure of 2, it might be expected that its amine-terminated sidearm might enhance its ability to chelate metals because the oxygen atom (and possibly the nitrogen atom) in the new side-arm of the molecule could wrap around and help stabilize metal atoms that would bind to the crown. To within experimental error the PDADMAC and PSS-2 films in the PDADMAC/PSS-2 bilayer had the same ellipsometric thicknesses as the PDADMAC and PSS-1 films in the PDADMAC/PSS-1 bilayer (see Table 1). Nevertheless, TOF-SIMS showed some significant and interesting differences between the films containing 1 and 2. First, there is essentially no enhancement of the (M $+1)^{+}$signal in the PDADMAC/PSS-2 bilayer compared to the signal from 2 dissolved in $\mathrm{HCl}$ and dried on a surface, although both of these signals were much larger than the essentially nonexistent signal of $(\mathrm{M}+1)^{+}$from neat 2 (see Figure 8a). However, unlike the PDADMAC/ PSS-1 bilayer, the PDADMAC/PSS-2 surface showed a significant amount of $\mathbf{2}$ that is cationized by $\mathrm{Na}^{+}$(Figure $8 \mathrm{~b})$ and $\mathrm{K}^{+}$(Figure 8c). Theoretical (see insets) and experimental isotope patterns are again in essentially exact agreement. As Figure 8 shows, the signals of cationized 2 are much stronger than those from the controls. No sign of 1 cationized by an alkali metal was seen from either the bilayer or the controls. $\mathrm{K}^{+}$is presumably an impurity in the system that is strongly complexed by 2 .

It is of interest to know whether the same ion yields observed in this work can be produced simply by mixing the analyte with a polyelectrolyte in solution and then allowing a droplet of the resulting solution to dry on a surface. To test this possibility, we made two solutions using analytes 3 and 4 mixed with PSS and
PDADMAC, respectively, and then allowed droplets of them to dry on a surface. Results of this study are shown in Table 2. For 4, the largest TOF-SIMS signals are obtained from the PDADMAC-4 monolayer and the powder sample. However, the resolution of the signal from the powder is very poor. Compared with the signal from 4 dissolved in dilute $\mathrm{NH}_{4} \mathrm{OH}$, the droplet from a solution of 4 and PDADMAC shows a reasonable enhancement in the analyte signal, but it is still much lower than the signal from the PDADMAC-4 monolayer. For 3, the best results in terms of signal and high-resolution are obtained for the PDADMAC/PSS-3 bilayer. The simple mixture of PSS and 3 that was allowed to dry on a surface shows about half of the intensity of the bilayer. The powder 3 sample and the sample of 3 dissolved in dilute $\mathrm{HCl}$ show smaller, but still significant signals. Thus, apparently simple mixtures of polyelectrolytes and analytes do show enhancements in ion yield, but not to the degree that the ultra-thin polyelectrolyte mono- and bilayers do.

The mechanism for organic ion production in SIMS is not fully understood. Nevertheless, one can speculate about the reasons for the enhancements in ion yields and cationized species in this work. The yields may be a result of the highly ionic nature of the PE matrix that the analytes find themselves in. Indeed, it is of interest that most of the counterions of PEs in solution are absent in the final PE multilayers [10]. If the analytes considered in this work behave similarly, then they would exist in the bi- or monolayers as ionized species with enormous counterions. Certain conformations of the PEs, including those that may be induced by primary ion bombardment, may weaken their ionic bonds to the analytes. Not all analytes show the enhancements reported in this work, e.g., no signal from $\mathbf{6}$ is observed from a PDADMAC-6 monolayer, possibly because of its high water solubility. Nevertheless, the enhancements in ion yields and/or cationization that have been observed for a number of different analytes at low surface concentrations suggests that this method merits further study and that it may become an effective analytical tool. 


\section{Acknowledgments}

The authors acknowledge the Department of Chemistry and Biochemistry and the College of Physical and Mathematical Sciences at Brigham Young University for their support of this work.

\section{References}

1. TOF-SIMS, Surface Analysis by Mass Spectrometry; Vickerman, J. C.; Briggs, D., Eds.; IM Publications: Chichester, UK, 2001; pp 753-778.

2. Hagenhoff, B. Optimization Methods: Cationization. In TOFSIMS, Surface Analysis by Mass Spectrometry; pp Vickerman, J. C.; Briggs, D., Eds.; IM Publications: Huddersfield, UK, 2001; 285-308.

3. Benninghoven, A.; Sichtermann, W. Secondary Ion Mass Spectrometry: A New Analytical Technique for Biologically Important Compounds. Org. Mass Spectrometry. 1977, 12, 595-597.

4. Michel, R.; Luginbühl, R.; Graham, D. J.; Ratner, B. D. New Substrates for Polymer Cationization with Time-of-Flight Secondary Ion Mass Spectrometry. Langmuir 2000, 16, 6503-6509.

5. Benninghoven, A.; Rudenauer, F. G.; Werner, H. W. Secondary Ion Mass Spectrometry; John Wiley and Sons: New York, 1987.

6. Grade, H.; Winograd, N.; Cooks, R. G. Cationization of Organic Molecules in Secondary Ion Mass Spectrometry. J. Am. Chem. Soc. 1977, 99, 7725-7726.

7. Gusev, A. I.; Choi, B. K.; Hercules, D. M. Improvement of Signal Intensities in Static Secondary-Ion Mass Spectrometry Using Halide Additives and Substrate Modification. J. Mass Spectrom. 1998, 33, 480-485.

8. Delcorte, A.; Médard, N.; Bertrand, P. Organic Secondary Ion Mass Spectrometry: Sensitivity Enhancement by Gold Deposition. Anal. Chem. 2002, 74, 4955-4968.

9. Wojciechowski, I.; Delcorte, A.; Gonze, X.; Bertrand, P. Mechanism of Metal Cationization in Organic SIMS. Chem. Phys. Lett. 2001, 346, 1-8.

10. Decher, G. Fuzzy Nanoassemblies: Toward Layered Polymeric Multicomposites. Science 1997, 277, 1232-1237.

11. Delcorte, A.; Bertrand, P.; Arys, X.; Jonas, A.; Wischerhoff, E.; Mayer, B.; Laschewsky, A. TOF-SIMS Study of Alternate Polyelectrolyte Thin Films: Chemical Surface Characterization and Molecular Secondary Ions Sampling Depth. Surf. Sci. 1996, $366,149-165$.

12. Delcorte, A.; Bertrand, P.; Wischerhoff, E.; Laschewsky, A. Adsorption of Polyelectrolyte Multilayers on Polymer Surfaces. Langmuir 1997, 13, 5125-5136.

13. Laschewsky, A.; Wischerhoff, E.; Bertrand, P.; Delcorte, A. Polyelectrolyte Multilayers Containing Photoreactive Groups. Macromol. Chem. Phys. 1997, 198, 3239-3253.

14. Cooper, T. M.; Campbell, A. L.; Crane, R. L. Formation of Polypeptide-Dye Multilayers by Electrostatic Self-Assembly Technique. Langmuir 1995, 11, 2713-2718.

15. Ariga, K.; Lvov, Y.; Kunitake, T. Assembling Alternate DyePolyion Molecular Films by Electrostatic Layer-by-Layer Adsorption. J. Am. Chem. Soc. 1997, 119, 2224-2231.

16. Linford, M. R.; Auch, M.; Möhwald, H. Nonmonotonic Effect of Ionic Strength on Surface Dye Extraction During DyePolyelectrolyte Multilayer Formation. J. Am. Chem. Soc. 1998, 120, 178-182.

17. Ariga, K.; Onda, M.; Lvov, Y.; Kunitake, T. Alternate Layer-by-Layer Assembly of Organic Dyes and Proteins is Facilitated by Premixing with Linear Polyions. Chem. Lett. 1997, 25-26.

18. Dawydoff, W.; Linow, K.-J.; Philipp, B. Acta Polym. 1991, 42, 592-596.

19. Dawydoff, W.; Linow, K.-J.; Philipp, B. Acta Polym. 1991, 42, 646-650.

20. Lua, Y.-Y.; Fillmore, W. J. J.; Linford, M. R. Aldehydes React with Scribed Silicon to Form Alkyl Monolayers. Characterization by TOF-SIMS Suggests an Even-Odd Effect. Appl. Surf. Sci. 2004, 231/232, 323-327.

21. Prodi, L.; Montalti, M.; Zaccheroni, N.; Bradshaw, J. S.; Izatt, R. M.; Savage, P. B. Characterization of 5-Chloro-8 Methoxyquinoline Appended Diaza-18-Crown-6 as a Chemosensor for Cadmium. Tetrahedron Lett. 2001, 42, 2941-2944.

22. Bain, C. D.; Whitesides, G. M. Attenuation Lengths of Photoelectrons in Hydrocarbon Films. J. Phys. Chem. 1989, 93, 1670-1673.

23. Moulder, J. F.; Stickle, W. F.; Sobol, P. E.; Bomben, K. D. Handbook of X-ray Photoelectron Spectroscopy; Physical Electronics, Inc.: Eden Prarie, MN, 1995. 